\title{
A hybrid Jaya Algorithm for Reliability-Redundancy Allocation Problems
}

\author{
Sahand Ghavidel ${ }^{\mathrm{a}}$, Ali Azizivahed ${ }^{\mathrm{b}}, \mathrm{Li} \mathrm{Li}^{\mathrm{a}}$ \\ ${ }^{\text {a}}$ Faculty of Engineering and Information Technology, University of Technology, \\ Sydney, PO Box 123, Broadway, NSW 2007, Australia \\ ${ }^{\mathrm{b}}$ Department of Electrical and Electronics Engineering, Shiraz University of Technology, \\ Shiraz, Iran \\ *Corresponding Author: S. Ghavidel, Faculty of Engineering and Information Technology, \\ University of Technology, Sydney, PO Box 123, Broadway, NSW 2007, Australia (e-mail: \\ sahand.ghavideljirsaraie@ @student.uts.edu.au).
}

\begin{abstract}
:
This paper proposes an efficient improved hybrid Jaya algorithm based on TimeVarying Acceleration Coefficients (TVAC) and learning phase introduced in TeachingLearning-Based Optimization (TLBO), named LJaya-TVAC algorithm, for solving various types of nonlinear mixed-integer Reliability-Redundancy Allocation Problems (RRAPs) and standard real-parameter test functions. RRAPs include series, series-parallel, complex (bridge) and overspeed protection systems. The search power of proposed LJaya-TVAC algorithm for finding the optimal solutions is firstly tested on the standard real-parameter unimodal and multi-model functions with dimension of 30 to 100 , and then tested on various types of nonlinear mixed-integer RRAPs. The results are compared with the original Jaya algorithm and best results reported in the recent literature. The obtained optimal results of proposed LJaya-TVAC algorithm provide evidence for the better and acceptable optimization performance compared to the original Jaya algorithm and other reported optimal results.
\end{abstract}


Keywords: Jaya algorithm, Reliability-Redundancy Allocation Problems (RRAPs), Teaching-Learning-Based Optimization (TLBO) algorithm, Time-Varying Acceleration Coefficients.

Nomenclature:

\begin{tabular}{|c|c|c|c|}
\hline$C$ & upper limit on the cost of the system & $R_{s}$ & reliability of the system \\
\hline$f(.,)$. & objective function of reliability of the system & $r_{d}(d=1: m)$ & $\begin{array}{l}\text { reliability of every component } \\
\text { available for the } d^{\text {th }} \text { subsystem }\end{array}$ \\
\hline$g(.,)$. & set of constraint functions & $T$ & operating time of problem 4 \\
\hline$m$ & number of subsystems in the system & $V$ & $\begin{array}{l}\text { upper limit on the sum of the } \\
\text { subsystems' products of volume }\end{array}$ \\
\hline $\begin{array}{l}n_{d} \\
(d=1: m)\end{array}$ & number of components in the $d^{\text {th }}$ subsystem & $V_{1}$ to $V_{4}$ & control valves of problem 4 \\
\hline$P 1$ & problem 1 or series test system & $v_{d}$ & $\begin{array}{l}\text { volume of each component in the } d^{\text {th }} \\
\text { subsystem }\end{array}$ \\
\hline$P 2$ & problem 2 or series-parallel test system & $W$ & $\begin{array}{l}\text { upper limit on the weight of the } \\
\text { system }\end{array}$ \\
\hline$P 3$ & problem 3 or complex test system & $w_{d}$ & $\begin{array}{l}\text { weight of each component in the } d^{\text {th }} \\
\text { subsystem }\end{array}$ \\
\hline$P 4$ & $\begin{array}{l}\text { problem } 4 \text { or overspeed protection test } \\
\text { system }\end{array}$ & $Z^{+}$ & $\begin{array}{l}\text { set of positive integers in the discrete } \\
\text { space }\end{array}$ \\
\hline$P 5$ & problem 5 or large-scale test system & $\alpha_{d}$ and $\beta_{d}$ & $\begin{array}{l}\text { physical characteristics of the system } \\
\text { components }\end{array}$ \\
\hline $\begin{array}{l}q_{d} \\
(d=1: m)\end{array}$ & $\begin{array}{l}\text { failure probability of each component in the } \\
d^{\text {th }} \text { subsystem }\end{array}$ & & \\
\hline $\begin{array}{l}R_{d} \\
(d=1: m)\end{array}$ & reliability of the $d^{\text {th }}$ subsystem & & \\
\hline
\end{tabular}




\section{Introduction}

The main goal of maximizing the Reliability-Redundancy Allocation Problems (RRAPs) includes a selection of the levels and redundancy of the components to maximize and improve the system reliability and performance. The RRAPs are beneficial for the design of the systems that are brought together on a large scale and produced in a large-scale industrial operation using off-the-shelf components. These days, as a consequence of increasing system complications and unpredictable behaviors, evaluating the reliability of the systems and the requirement for improving the reliability of the systems have become very interesting and significant (Zhang et al. 2013).

Various optimization programming and evolutionary techniques have been employed to optimize various types of RRAPs, such as: Genetic Algorithm (GA) (Hsieh, Chen, and Bricker 1998; He et al. 2013), Particle Swarm Optimization (PSO) (Wu et al. 2011; Zhang et al. 2013; Tan, Tan, and Deng 2013), Simulated Annealing (SA) (Dohi et al. 2006; Suman 2003), Harmony Search (HS) (Zou et al. 2010; Zou et al. 2011; dos Santos Coelho, Diego, and Mariani 2011), Tabu Search (TS) (Jang and Kim 2011; Liu and Qin 2014), a coldstandby redundancy strategy (Ardakan and Hamadani 2014a), a combination search algorithm based on Hooke-Jeeves pattern search and dynamic programming (Liu 2006), the RRAP of parking facilities in the real system using a hybrid GA (Hamadani et al. 2013), Ant Colony Optimizer (ACO) (Liang and Smith 2004), Cuckoo Search (CS) (Valian and Valian 2013; Valian et al. 2013), Memetic Algorithm (MA) (Pourdarvish and Ramezani 2013), Imperialist Competitive Algorithm (ICA) (Afonso, Mariani, and dos Santos Coelho 2013), Artificial Bee Colony (ABC) (Yeh and Hsieh 2011), a hybrid algorithm of space partitioning and tabu-genetic (SP/TG) (Ouzineb, Nourelfath, and Gendreau 2011) for non-homogeneous RRAP, Honey Bee Mating Optimization (HBMO) (Sadjadi and Soltani 2012), a new mixed 
strategy which uses cold-standby and active strategies with a proposed GA for reliability optimization of series-parallel systems (Ardakan and Hamadani 2014b), compromise programming (Soltani, Sadjadi, and Tavakkoli-Moghaddam 2015), binary equivalent models and Mixed Integer Nonlinear Programming (MINLP) for the cold standby RRAP (Feizollahi, Soltani, and Feyzollahi 2015), Immune Algorithm (IA) (Chen and You 2005; Chen 2006), a multi-objective multi-stage reliability growth planning strategy (Li, Mobin, and Keyser 2016) using a modified non-dominated sorting GA (NSGA-II) in the early product-development stage and also multi-objective reliability optimization using GA proposed by (Ardakan, Hamadani, and Alinaghian 2015), Improved Bat Algorithm (IBA) (Liu 2016), neighbourhood search heuristic method with nonlinear programming (Chatwattanasiri, Coit, and Wattanapongsakorn 2016), and a Penalty Guided Stochastic Fractal Search (PSFS) (Mellal and Zio 2016), a new interpretation and formulation of the RRAP (Ardakan Abouei et al. 2016) using the mixed new strategy and a modified version of the GA (MVGA), showing distinct advantages compared to traditional methods, and etc. A state of the art survey of optimization techniques for various types of RRAP to 2014 is presented in (Soltani 2014).

Jaya algorithm (Rao 2016) is a new simple and efficient algorithm. Similar to the other algorithms, it only has the common parameters that will be determined by the user like population number and iterations of algorithm without need of any specific control parameters that would be determined by the user. This algorithm is based on the best and the worst candidate solutions in the iterations (Rao 2016). It has good feasibility and performance in solving different engineering optimization problems such as complex constrained design optimization (Venkata Rao and Waghmare 2016), dimensional optimization of a microchannel heat sink (Rao et al. 2016), and surface grinding process optimization (Rao, Rai, and Balic 2016). To the authors' best knowledge, this is the first time that the Jaya algorithm is 
used for RRAPs in this study.It can be shown that the results obtained by Jaya algorithm for RRAPs are suitable and good.

RRAPs are an important requirement of various systems. In many systems, balance in weights, number of components in subsystems and/or low cost are desired. In various cases, the methods and optimization algorithms described provide solutions very close to optimality for RRAPs of various systems. In this study, an improved new optimization algorithm has been presented to meet these requirements to solve various RRAPs. This paper proposes a hybrid enhanced Jaya algorithm based on the learning phase of TeachingLearning-Based Optimization (TLBO) algorithm introduced in (Rao, Savsani, and Vakharia 2011; Rao 2015; Rao and Patel 2012) with its applications (Rao 2015; Ghasemi 2014, 2015), and a new Time-Varying Acceleration Coefficients (TVAC) proposed by (Ratnaweera, Halgamuge, and Watson 2004) for solving various types of nonlinear mixed-integer RRAPs. In the first phase of proposed Jaya-TVAC algorithm, a TVAC is added to the Jaya algorithm, and then in the second phase, a learning phase of the TLBO algorithm (Rao, Savsani, and Vakharia 2011; Rao 2015; Rao and Patel 2012) is added to the Jaya-TVAC algorithm (LJayaTVAC algorithm) for finding the better final solutions with higher convergence rate compared with the original algorithm. The two new time-varying acceleration coefficients added to the Jaya algorithm increase the search power around the global optimal solution in the primary iterations for faster convergence. The added learning phase also increases the search power in the final iterations for finding the better final solutions with higher convergence rate through the increased local search of Jaya.

This study is arranged as follows: Section 2 provides a formulation and description of the RRAPs for test systems such as series, series-parallel, complex and overspeed protection system. In Sections 3 and 4, the Jaya and hybrid enhanced Jaya algorithms using TVAC and learning phase are presented. Section 5 shows performance of the proposed optimization 
algorithms in solving RRAPs for the various systems and also standard real-parameter test functions. We end this study with some conclusions for the hybrid enhanced Jaya algorithm in Section 6.

\section{Reliability-Redundancy Allocation Problems (RRAPs)}

The main purpose of optimization of the RRAPs is to enhance the reliability of these systems (maximization of the overall system reliability) by means of using component reliabilities allocation $\left(r=\left(r_{1}, r_{2}, \ldots, r_{m}\right)\right)$ and redundancy allocation number $\left(n=\left(n_{1}, n_{2}, \ldots, n_{m}\right)\right)$. The nonlinear mixed-integer programming model of these problems can be formulated by maximizing the reliability of the system as the objective function subject to multiple nonlinear constraints as the following equations:

Maximize $R_{s}=f(r, n)$,

subject to $g(r, n) \leq l$

$$
0 \leq r_{d} \leq 1, n_{d} \in Z^{+}, 0 \leq d \leq m .
$$

where $R_{S}$ is the reliability of the system, $f(.,$.$) and g(.,$.$) are the objective function and$ constraints of the RRAPs, respectively; $g($.$) is usually associated with the system cost,$ volume and weight limitations. $r=\left(r_{1}, r_{2}, \ldots, r_{m}\right)$ and $n=\left(n_{1}, n_{2}, \ldots, n_{m}\right)$ are the component reliabilities and redundancy allocation number vectors for $m$ subsystems, and also, $l$ is the system resource limitation.

Four RRAPs including the series system (problem $1(P 1)$ ), series-parallel system (problem 2 $(P 2)$ ), complex (bridge) system (problem $3(P 3)$ ) and overspeed protection system of a gas turbine (problem $4(P 4))$ are evaluated in this paper as follows.

\subsection{Series system $(P 1)$}


The series system with $m=5$ subsystems (for $d=1: m$ ) for nonlinear mixed-integer RRAP was presented in (Chen 2006; Hsieh, Chen, and Bricker 1998). The block diagram of the series system with five subsystems is shown in Fig. 1 (Afonso, Mariani, and dos Santos Coelho 2013). The RRAP of the series system can be formulated as follows (Chen 2006):

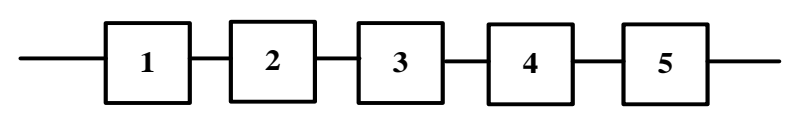

Fig. 1. Block diagram of the series system

$$
\begin{aligned}
\text { Maximize } & f_{1}(r, n)=\prod_{d=1}^{m} R_{d}\left(n_{d}\right) \\
0 \leq r_{d} \leq 1, & n_{d} \in Z^{+} \text {(positive integer in the discrete space). }
\end{aligned}
$$

Here, $R_{d}\left(n_{d}\right)=1-q_{d}^{n_{d}}$ is the reliability of $d^{\text {th }}$ subsystem, $q_{d}=1-r_{d}$ is the failure probability of each component in $d^{\text {th }}$ subsystem, and $n_{d}$ is the number of components in the $d^{\text {th }}$ subsystem. The objection function is subject to the following constraints:

1- The combination of weight, volume, and redundancy allocation number constraint $g_{1}(r, n):$

$g_{1}(r, n)=\sum_{d=1}^{m} w_{d} v_{d}^{2} n_{d}^{2} \leq V$

where, $w_{d}$ is the weight of each component in $d^{\text {th }}$ subsystem; $v_{d}$ is the volume of each component in $d^{\text {th }}$ subsystem, $V$ is the upper limit on the sum of the subsystems' products of volume.

2- The system cost constraint $g_{2}(r, n)$ :

$$
g_{2}(r, n)=\sum_{d=1}^{m} \alpha_{d}\left(-\frac{1000}{\ln r_{d}}\right)^{\beta_{d}}\left[n_{d}+e^{0.25 n_{d}}\right] \leq C
$$


where, $\alpha_{d}$ and $\beta_{d}$ are physical characteristics of the system components. Also, $C$ is the upper limit on the cost of the system.

3- The system weight constraint $g_{3}(r, n)$ :

$g_{3}(r, n)=\sum_{d=1}^{m} w_{d} n_{d} e^{0.25 n_{d}} \leq W$

where, $W$ is the upper limit on the weight of the system. The parameters of the series system (Chen 2006; Hsieh, Chen, and Bricker 1998) are given in Table A.1 in Appendix.

\subsection{Series-parallel system (P2)}

The series-parallel system $(P 2)$, with the same $g_{1}(r, n), g_{2}(r, n)$ and $g_{3}(r, n)$ constraints as those of $P 1$, is shown in Fig. 2 (Chen 2006; Hsieh, Chen, and Bricker 1998). The input parameters of this test system (P2) (Chen 2006; Hsieh, Chen, and Bricker 1998) are given in Table A.2 in Appendix.

The nonlinear mixed-integer RRAP of the series-parallel system can be formulated as follows (Dohi et al. 2006):

$$
\begin{aligned}
& \text { Maximize } f_{2}(r, n)=1-\left(1-R_{1} R_{2}\right)\left(1-\left(1-\left(1-R_{3}\right)\left(1-R_{4}\right)\right) R_{5}\right) \\
& \quad 0 \leq r_{d} \leq 1, n_{d} \in Z^{+} \text {(positive integer in the discrete space), } 1 \leq d \leq 5 .
\end{aligned}
$$

subject to $g_{1}(r, n), g_{2}(r, n)$ and $g_{3}(r, n)$.

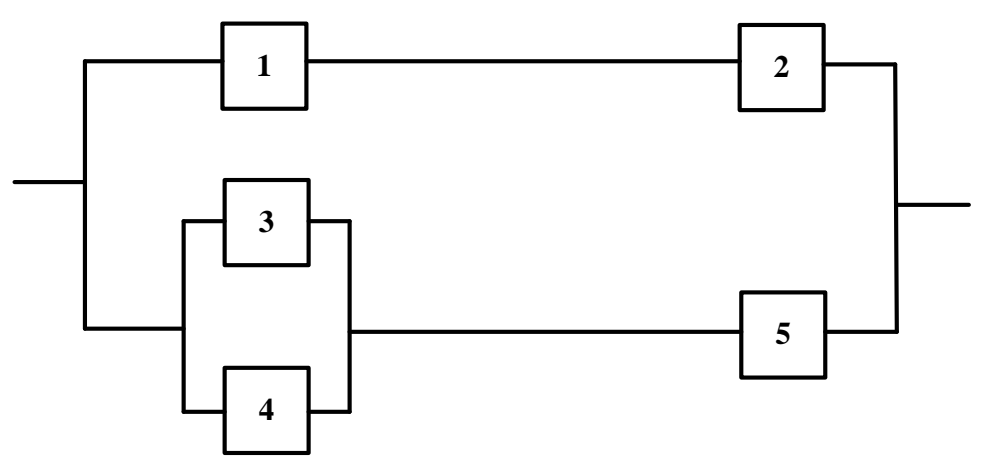


Fig. 2. Block diagram of the series-parallel system.

\subsection{Complex (bridge) system (P3)}

The complex (bridge) system (P3) is shown in Fig. 3 which has the same non-linear constraints $g_{1}(r, n), g_{2}(r, n)$ and $g_{3}(r, n)$ as those of the series and series-parallel systems optimization problems (Chen 2006). The input parameters of this test system (P3) (Chen 2006; Hsieh, Chen, and Bricker 1998) are given in Table A.3 in Appendix.

The nonlinear mixed-integer RRAP of the complex (bridge) system $(P 3)$ can be formulated as follows:

Maximize $f_{3}(r, n)=R_{1} R_{2}+R_{3} R_{4}+R_{1} R_{4} R_{5}+R_{2} R_{3} R_{5}-R_{1} R_{2} R_{3} R_{4}-R_{1} R_{2} R_{3} R_{5}$

$-R_{1} R_{2} R_{4} R_{5}-R_{1} R_{3} R_{4} R_{5}-R_{2} R_{3} R_{4} R_{5}+2 R_{1} R_{2} R_{3} R_{4} R_{5}$

$$
0 \leq r_{d} \leq 1, n_{d} \in Z^{+}, 1 \leq d \leq 5 \text {. }
$$

subject to $g_{1}(r, n), g_{2}(r, n)$ and $g_{3}(r, n)$.

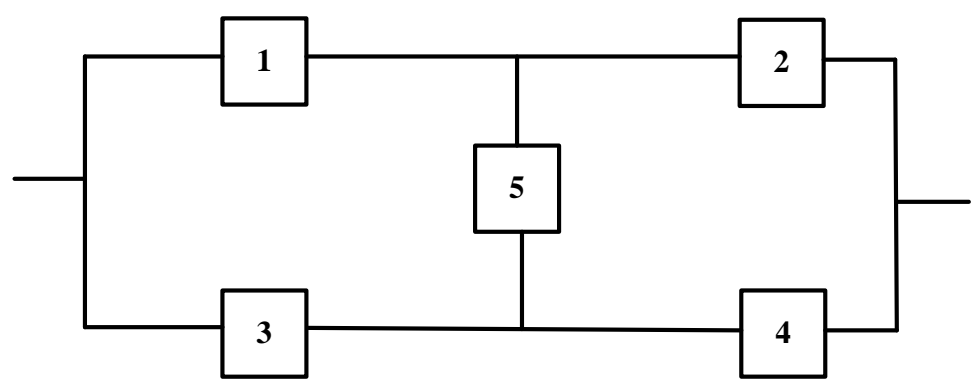

Fig. 3. Block diagram of the complex (bridge) system.

\subsection{Overspeed protection system of a gas turbine $(P 4)$}

The overspeed detection is constantly supplied by the mechanical and electrical systems. When an overspeed happens, it is essential to stop the fuel source by means of using control valves $\left(V_{1}\right.$ to $\left.V_{4}\right)$. The overspeed protection system of a gas turbine for the fourth nonlinear 
mixed-integer RRAP is shown in Fig. 4. The input parameters of the overspeed protection system (Chen 2006) are given in Table A.4 in Appendix.

This RRAP of the overspeed protection system a gas turbine can be formulated as follows:

$$
\begin{gathered}
\text { Maximize } f_{4}(r, n)=\prod_{d=1}^{m}\left[1-\left(1-r_{d}\right)^{n_{d}}\right] . \\
0.5 \leq r_{d} \leq\left(1-10^{-6}\right), \\
1 \leq n_{d} \leq 10, n_{d} \in Z^{+} .
\end{gathered}
$$

This objective function is subject to the following constraints:

1- The combination of weight, volume, and redundancy allocation number constraint

$$
g_{1}(r, n):
$$

$g_{1}(r, n)=\sum_{d=1}^{m} v_{d}^{2} n_{d}^{2} \leq V$

2- The system cost constraint $g_{2}(r, n)$ :

$$
\begin{aligned}
& g_{2}(r, n)=\sum_{d=1}^{m} C\left(r_{d}\right)\left[n_{d}+e^{0.25 n_{d}}\right] \leq C, \\
& C\left(r_{d}\right)=\alpha_{d}\left(-\frac{T}{\ln r_{d}}\right)^{\beta_{d}} .
\end{aligned}
$$

where, $C\left(r_{d}\right)$ is the cost of each component with reliability $r_{d}$ at the $d^{\text {th }}$ subsystem, and $T$ is the operating time in which the component must not fail.

3- The system weight constraint $g_{3}(r, n)$ :

$$
g_{3}(r, n)=\sum_{d=1}^{m} w_{d} n_{d} e^{0.25 n_{d}} \leq W
$$




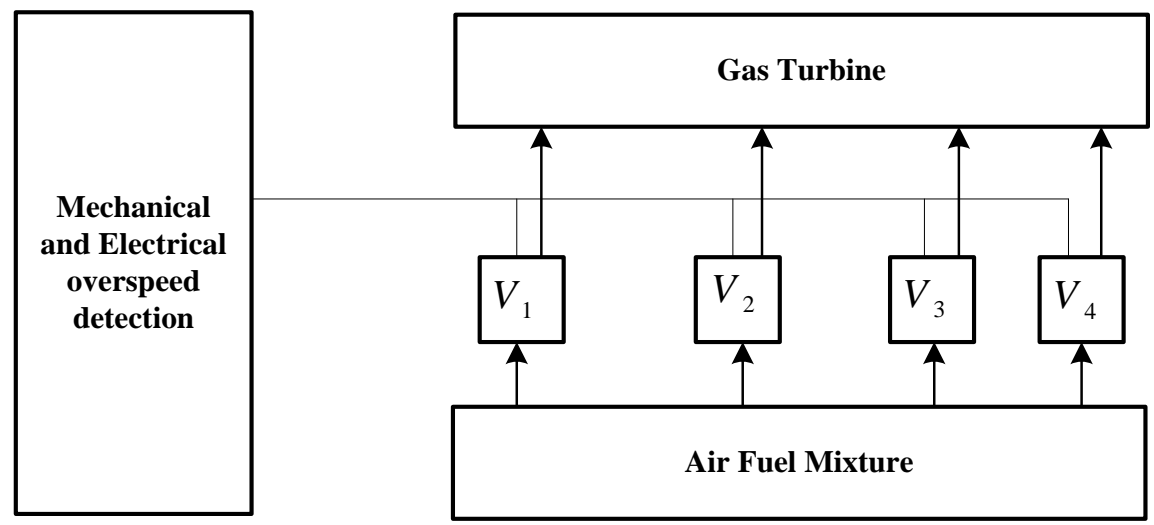

Fig. 4. The diagram block for the overspeed protection system of a gas turbine.

\subsection{Large scale RRAP (P5):}

To clearly show the effectiveness of the proposed LJaya-TVAC algorithm for RRAP, a large scale system (Zhang et al. 2013) is used with the same non-linear constraints $g_{1}(r, n)$, $g_{2}(r, n)$ and $g_{3}(r, n)$ as those of the overspeed protection test system (Mellal and Zio 2016). The formulation of this problem can be written as follows (Zhang et al. 2013):

$$
\begin{gathered}
\text { Maximize } f_{5}(r, n)=\prod_{d=1}^{m}\left[1-\left(1-r_{d}\right)^{n_{d}}\right] . \\
0.5 \leq r_{d} \leq\left(1-10^{-6}\right), m=20, \\
1 \leq n_{d} \leq 10, n_{d} \in Z^{+} .
\end{gathered}
$$


The large-scale test system includes forty decisions variables $(=m * 2=40)$. The data and input parameters for the large-scale test system are given in (Zhang et al. 2013).

\section{Jaya algorithm}

Jaya algorithm (Rao 2016) is a recently proposed algorithm which is a powerful and simple optimizer for real-world optimization problems. The original flowchart of the optimization process for Jaya algorithm is shown in Fig. 5 (Rao 2016). In the Jaya algorithm, each member of all population $(N)$, has its own location (solution) in the $i^{\text {th }}$ iteration $\left(i=1: i_{\max }\right)$ of the algorithm. $X_{k}^{i}(k=1: N)$ is defined by the optimization problem parameters in the $d$ dimensional solution search space: $X_{k}^{i}=\left[x_{1, k}^{i}, x_{2, k}^{i}, \ldots, x_{d, k}^{i}\right]$. The new location value $X_{k}^{i+1}=\left[x_{1, k}^{i+1}, x_{2, k}^{i+1}, \ldots, x_{d, k}^{i+1}\right]$ for the $k^{\text {th }}$ member $X_{k}^{i}$ is achieved by updating the locations iteratively. If $f\left(X_{k}^{i+1}\right) \leq f\left(X_{k}^{i}\right)$, the new location value $\left(X_{k}^{i+1}\right)$ replaces the old location value $\left(X_{k}^{i}\right)$ using the following equation (Rao 2016):

$X_{k}^{i+1}=X_{k}^{i}+\operatorname{rand}_{1}^{i}\left(X_{\text {best }}^{i}-\left|X_{k}^{i}\right|\right)-\operatorname{rand}_{2}^{i}\left(X_{\text {worst }}^{i}-\left|X_{k}^{i}\right|\right)$

where, $X_{\text {best }}^{i}=\left[x_{1, \text { best }}^{i}, x_{2, \text { best }}^{i}, \ldots, x_{d \text {, best }}^{i}\right]$ and $X_{\text {worst }}^{i}=\left[x_{1, \text { worst }}^{i}, x_{2, \text { worst }}^{i}, \ldots, x_{d, \text { worst }}^{i}\right]$ are the best and worst solutions obtained until the $i^{\text {th }}$ iteration of the algorithm, respectively. $\operatorname{rand}_{1}^{i}=\left[\operatorname{rand}_{1,1}^{i}, \operatorname{rand}_{1,2}^{i}, \ldots, \operatorname{rand}_{1, d}^{i}\right]$ andare two set of $\operatorname{rand}_{2}^{i}=\left[\operatorname{rand}_{2,1}^{i}, \operatorname{rand}_{2,2}^{i}, \ldots, \operatorname{rand}_{2, d}^{i}\right]$ random numbers in the range $[0,1]$ in the $i^{\text {th }}$ iteration of the algorithm. Also, $\left|X_{k}^{i}\right|$ is the absolute value of $X_{k}^{i}$. 


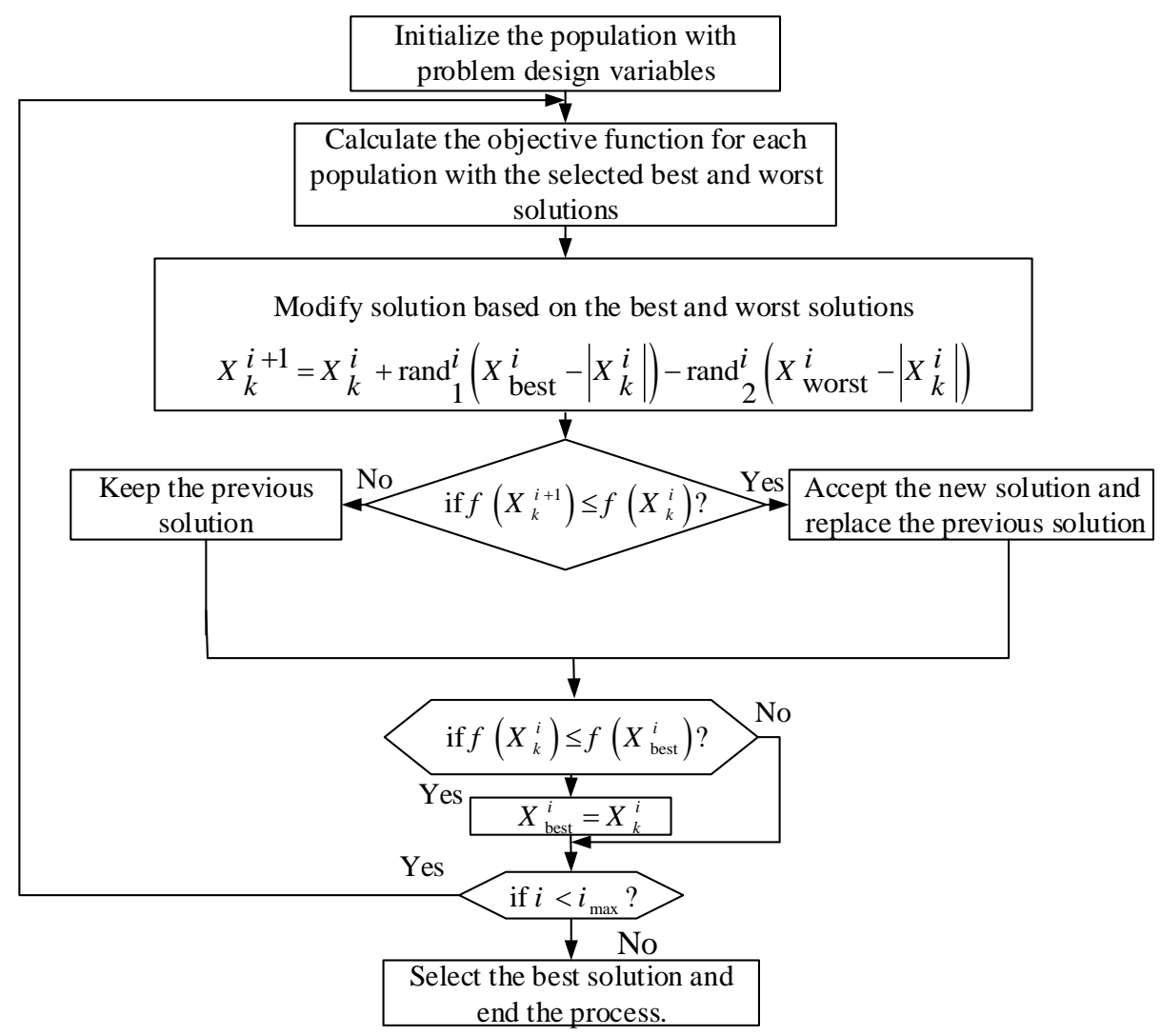

Fig. 5. The optimization process of the original Jaya algorithm.

\section{The hybrid enhanced Jaya algorithm}

In this section, the hybrid enhanced Jaya algorithms using TVAC and learning phase is presented. These algorithms increase the search power around the global optimal solution ( $\left.X_{\text {best }}\right)$ in the primary iterations for faster convergence, and also increase the search power in the latest iterations.

\subsection{Jaya algorithm with time-varying acceleration coefficients (Jaya-TVAC)}

In the first phase, two new time-varying acceleration coefficients $c_{1}^{i}$ and $c_{2}^{i}$ are proposed based on the method by (Ratnaweera, Halgamuge, and Watson 2004) to improve the Jaya algorithm, which is called Jaya-TVAC algorithm. The new location value for $X_{k}^{i}$ is then modified as follows: 


$$
\begin{aligned}
& X_{k}^{i+1}=X_{k}^{i}+c_{1}^{i} \times \operatorname{rand}_{1}^{i}\left(X_{\text {best }}^{i}-\left|X_{k}^{i}\right|\right)-c_{2}^{i} \times \operatorname{rand}_{2}^{i}\left(X_{\text {worst }}^{i}-\left|X_{k}^{i}\right|\right) \\
& c_{1}^{i}=c_{1}^{i=1}-\left(c_{1}^{i=1}-c_{1}^{i=i_{\max }}\right)\left(\frac{i}{i_{\text {max }}}\right) \\
& c_{2}^{i}=c_{2}^{i=i_{\max }}-\left(c_{2}^{i=i_{\max }}-c_{2}^{i=1}\right)\left(\frac{i_{\max }-i}{i_{\max }}\right)
\end{aligned}
$$

where, $c_{1}{ }^{i=1}=c_{2}{ }^{i=1}=1$ and $c_{1}^{i=i_{\max }}=0.5$ and $c_{2}^{i=i_{\max }}=0$ are obtained for the best values.

\subsection{Hybrid Jaya-TVAC algorithm with learning phase (LJaya-TVAC) based on TLBO algorithm}

In the second phase, a learning phase introduced in (Rao, Savsani, and Vakharia 2011; Rao 2015; Rao and Patel 2012) is added to the proposed algorithm for finding the better final solutions with higher convergence rate through increased local search of Jaya. The flowchart of the optimization process for LJaya-TVAC algorithm is shown in Fig. 6. The new location value $X_{k}^{i+1}$ can be achieved using (18). Here two solution variables $X_{j}^{i}$ ( $j^{\text {th }}$ member of the population) and $X_{h}^{i}$ ( $h^{\text {th }}$ member of the population) are randomly selected as shown in (18).

If the value of the objective function for the new location value $X_{k}^{i+1}$ is better than the old location value $X_{k}^{i}\left(f\left(X_{k}^{i+1}\right) \leq f\left(X_{k}^{i}\right)\right)$, the new location value $X_{k}^{i+1}$ will replace the old location value $X_{k}^{i}$.

$$
X_{k}^{i+1}=X_{k}^{i}+\operatorname{rand}_{3}^{i} \times\left\{\begin{array}{l}
X_{j}^{i}-X_{h}^{i} \text { if } f\left(X_{j}^{i}\right) \leq f\left(X_{h}^{i}\right) \\
X_{h}^{i}-X_{j}^{i} \text { else. }
\end{array}\right.
$$




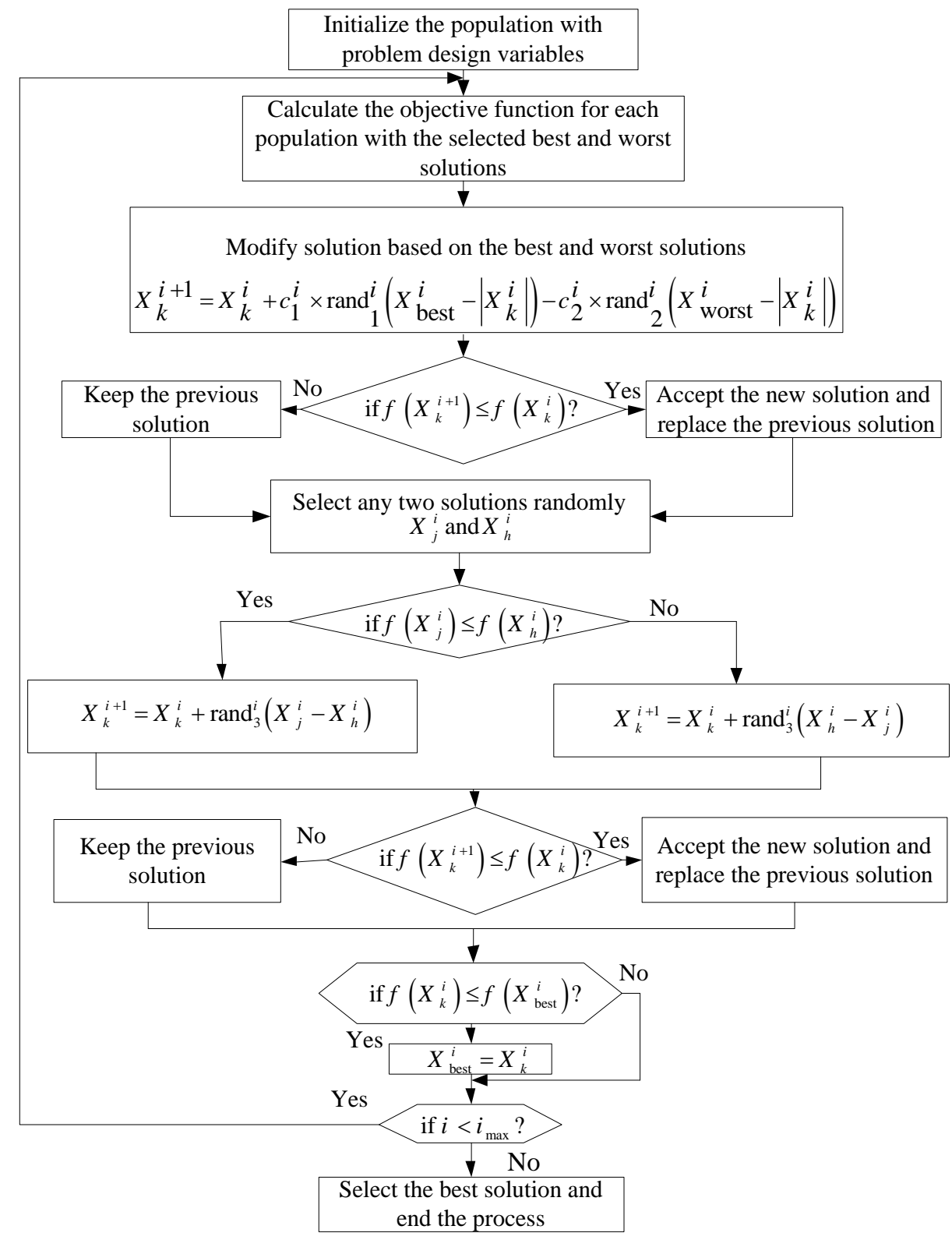

Fig. 6. The optimization process of LJaya-TVAC algorithm.

\section{Results and discussion}

\subsection{LJaya-TVAC algorithm for real-parameter problems}

In the first phase of the study, in order to validate the performance of the LJaya-TVAC algorithm for the real-parameter test functions, various types of real-parameter test functions are chosen (Suganthan et al. 2005). The details of sixth typical unimodal and multi-modal 
real-parameter test functions $(F)$ that are selected to evaluate the effectiveness of the proposed algorithms are summarized as follows:

1) $F_{1}$ : Shifted Rotated High Conditioned Elliptic (uni-modal, non-separable and scalable test function), $F_{1}(x)=\sum_{j=1}^{d}\left(10^{6}\right)^{\frac{j-1}{d-1}} z_{j}^{2}, z=(x-o) * M, x=\left[x_{1}, x_{2}, \ldots, x_{d}\right]$, with

$$
o=\left[o_{1}, o_{2}, \ldots, o_{d}\right]: \text { the shifted global optimum and, } M \text { : orthogonal matrix }
$$
$x_{j} \in[-100,100]$ and $F(x)=0$.

2) $F_{2}$ : Shifted Schwefel's Problem 1.2 with Noise in Fitness (uni-modal, non-separable and scalable test function), $F_{2}(x)=\left(\sum_{j=1}^{d}\left(\sum_{t=1}^{j} z_{t}\right)^{2}\right) *(1+0.4|N(0,1)|), z=x-o, o=\left[o_{1}, o_{2}, \ldots, o_{d}\right]:$ the shifted global optimum with $x_{j} \in[-100,100]$ and $F(x)=0$.

3) $F_{3}$ : Schwefel's Problem 2.6 with Global Optimum on Bounds (uni-modal, non-separable and scalable test function), $F_{3}(x)=\max \left\{\left|A_{j} x-B_{j}\right|\right\}$, $A$ is a $d * d$ matrix, $A_{j}$ is the $j$ th row of $A, B_{j}=A_{j} * o$.

with $x_{j} \in[-100,100]$ and $F(x)=0$.

4) $F_{4}$ : Shifted Rosenbrock's (multi-modal, non-separable and scalable test function), $F_{4}(x)=\sum_{i=1}^{d-1}\left(100\left(z_{j}^{2}-z_{j+1}\right)^{2}+\left(z_{j}-1\right)^{2}\right), z=x-o+1$. with $x_{j} \in[-100,100]$ and $F(x)=0$.

5) $F_{5}$ : Shifted Rotated Ackley's with Global Optimum on Bounds (multi-modal, nonseparable and scalable test function),

$$
F_{5}(x)=-20 \exp \left(-0.2 \sqrt{\frac{1}{d} \sum_{j=1}^{d} z_{j}^{2}}\right)-\exp \left(\frac{1}{d} \sum_{j=1}^{d} \cos \left(2 \pi z_{j}\right)\right)+20+e, z=x-o . \text { with } x_{j} \epsilon
$$

$[-32.0,32.0]$ and $F(x)=0$.

6) $F_{6}$ : Shifted Rastrigin's (multi-modal, separable and scalable test function), )$=0 . x(F 5.0,5.0]$ and $-\left[\epsilon_{j} x\right.$ with $F_{6}(x)=\sum_{j=1}^{d}\left(z_{j}^{2}-10 \cos \left(2 \pi z_{j}\right)+10\right), z=x-o$.

The Mean (mean value of the best results) and Std (standard deviation of the best results) indexes for the proposed Jaya algorithms of each real-parameter problems over 30 runs for $d=30$ and $d=100$ with $i_{\max }=d^{*} 1000$, and the population sizes of $N=50$ are given in Table 1 . Also Fig. 7 shows the convergence plots of the proposed Jaya algorithms for the real-parameter 
functions. The proposed LJaya-TVAC algorithm obtains better optimal results with faster convergence characteristics compared to the original Jaya and Jaya-TVAC algorithms. The results show that the proposed LJaya-TVAC method has been successfully implemented to the real-parameter optimization problems with different dimensions.

Table 1. The best results (Mean \pm Std) obtained from the Jaya algorithms for real-parameter problems.

\begin{tabular}{|c|c|c|c|c|c|c|c|}
\hline$F$ & $d$ & Jaya & Rank & Jaya-TVAC & Rank & LJaya-TVAC & Rank \\
\hline \multirow{2}{*}{$F_{1}$} & 30 & $5.70 \mathrm{e}+07 \pm 8.64 \mathrm{e}+06$ & 3 & $2.91 \mathrm{e}+07 \pm 2.16 \mathrm{e}+06$ & 2 & $1.93 \mathrm{e}+04 \pm 1.42 \mathrm{e}+04$ & $\mathbf{1}$ \\
\hline & 100 & $1.29 \mathrm{e}+09 \pm 1.57 \mathrm{e}+08$ & 3 & $8.07 \mathrm{e}+08 \pm 1.13 \mathrm{e}+08$ & 2 & $1.76 \mathrm{e}+06 \pm 6.78 \mathrm{e}+05$ & 1 \\
\hline \multirow{2}{*}{$F_{2}$} & 30 & $1.26 \mathrm{e}+04 \pm 1.27 \mathrm{e}+03$ & 3 & $6.07 \mathrm{e}+03 \pm 5.32 \mathrm{e}+03$ & 2 & $9.98 \mathrm{e}+01 \pm 7.56 \mathrm{e}+01$ & 1 \\
\hline & 100 & $3.68 \mathrm{e}+05 \pm 2.31 \mathrm{e}+04$ & 3 & $4.42 \mathrm{e}+04 \pm 2.87 \mathrm{e}+04$ & 2 & $2.43 e+04 \pm 1.85 e+04$ & $\mathbf{1}$ \\
\hline \multirow{2}{*}{$F_{3}$} & 30 & $3.90 \mathrm{e}+03 \pm 2.45 \mathrm{e}+03$ & 3 & $4.77 \mathrm{e}+02 \pm 5.83 \mathrm{e}+02$ & 2 & $1.82 \mathrm{e}+02 \pm 1.90 \mathrm{e}+02$ & $\mathbf{1}$ \\
\hline & 100 & $3.86 \mathrm{e}+04 \pm 3.09 \mathrm{e}+03$ & 3 & $1.07 \mathrm{e}+03 \pm 4.65 \mathrm{e}+03$ & 2 & $9.30 \mathrm{e}+02 \pm 2.24 \mathrm{e}+03$ & 1 \\
\hline \multirow{2}{*}{$F_{4}$} & 30 & $8.19 \mathrm{e}+07 \pm 3.82 \mathrm{e}+07$ & 3 & $2.52 \mathrm{e}+07 \pm 8.14 \mathrm{e}+06$ & 2 & $3.29 \mathrm{e}+00 \pm 3.21 \mathrm{e}+00$ & $\mathbf{1}$ \\
\hline & 100 & $5.36 \mathrm{e}+09 \pm 1.03 \mathrm{e}+09$ & 3 & $1.89 \mathrm{e}+09 \pm 7.36 \mathrm{e}+08$ & 2 & $2.98 \mathrm{e}+00 \pm 1.25 \mathrm{e}+00$ & $\mathbf{1}$ \\
\hline \multirow{2}{*}{$F_{5}$} & 30 & $20.871 \pm 0.083$ & 3 & $20.818 \pm 0.044$ & 2 & $20.72 \pm 0.061$ & $\mathbf{1}$ \\
\hline & 100 & $21.271 \pm 0.027$ & 3 & $21.065 \pm 0.017$ & 2 & $20.85 \pm 0.012$ & 1 \\
\hline \multirow{2}{*}{$F_{6}$} & 30 & $200.50 \pm 7.166$ & 3 & $180.59 \pm 12.42$ & 2 & $72.41 \pm 5.45$ & $\mathbf{1}$ \\
\hline & 100 & $896.75 \pm 69.301$ & 3 & $854.71 \pm 38.59$ & 2 & $464.64 \pm 26.36$ & 1 \\
\hline
\end{tabular}




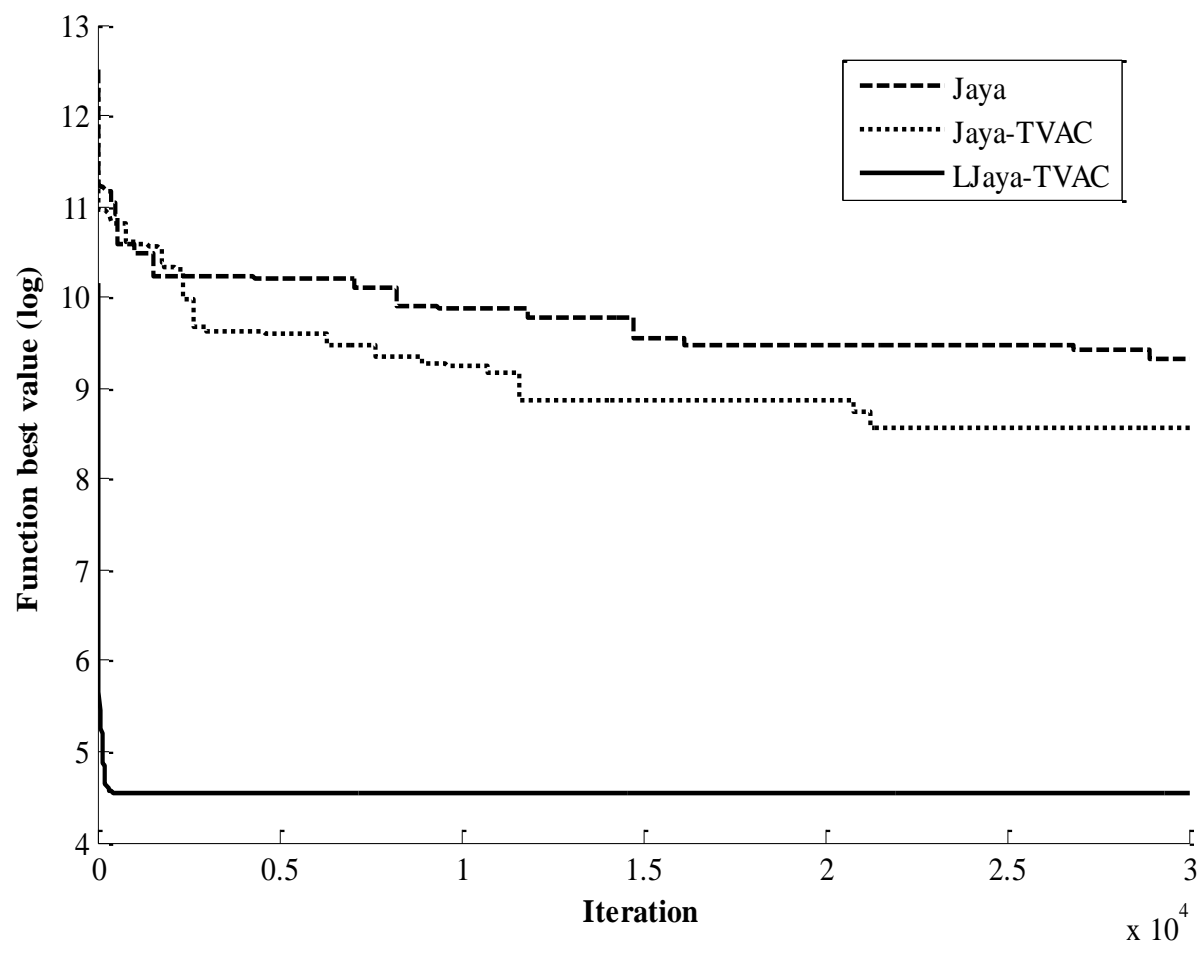

(a):

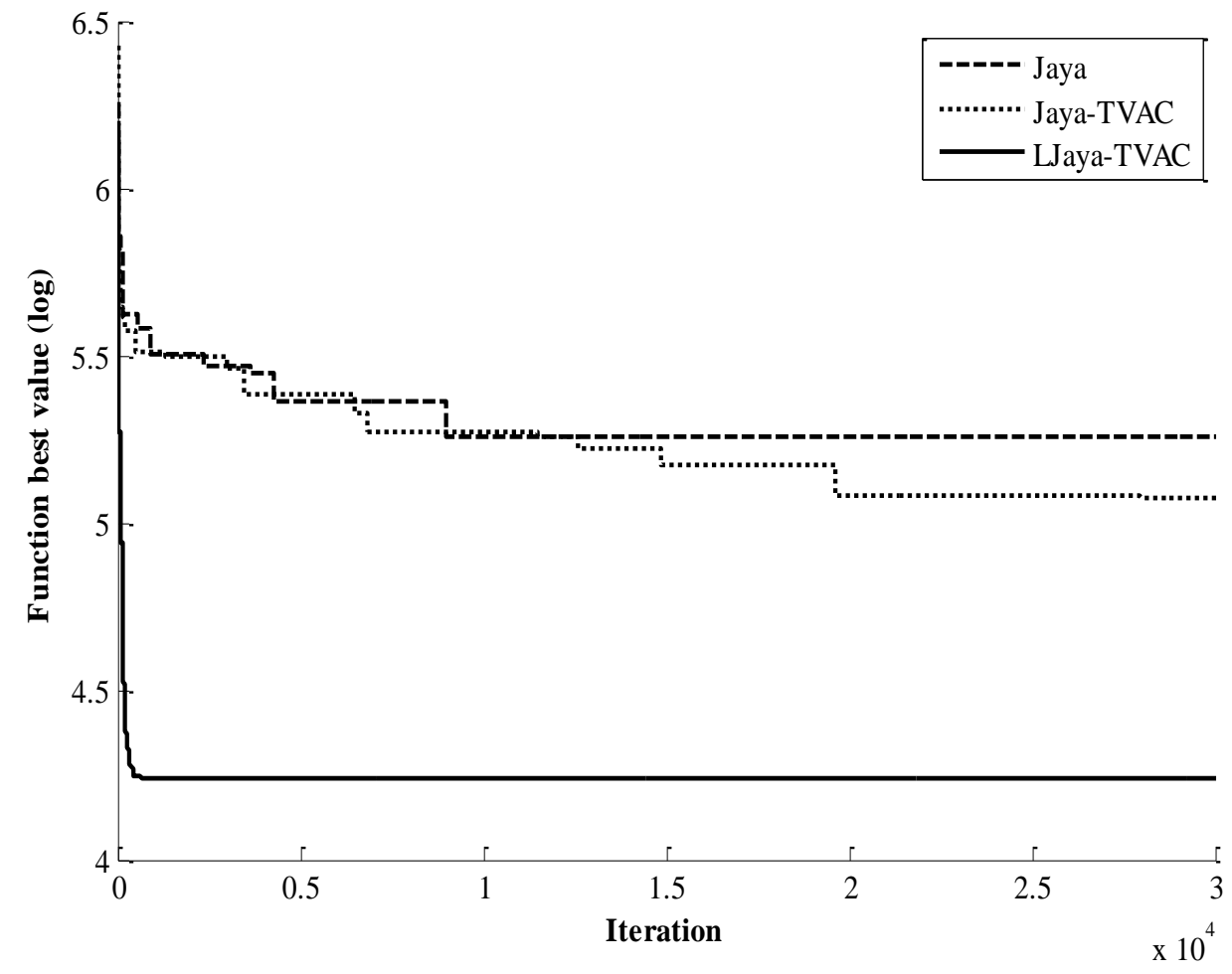

(b):

Fig. 7. Convergence plots of the proposed Jaya algorithms for the real-parameter function with $d=30$ : (a) $F_{2}$ and (b): $F_{6}$. 


\subsection{The optimization of RRAPs using LJaya-TVAC algorithm}

In the second study, the proposed LJaya-TVAC method is implemented for solving different RRAPs in various test systems. The optimization process of the proposed LJayaTVAC algorithm can be summarized as follows:

Step 1: Set the initial parameters $X_{d}^{\min }$ and $X_{d}^{\text {max }}$ (the minimum and maximum limits of variables for $\left.d=1: 2^{*} m\right), i_{\text {max }}, N, c_{1}^{i=1}=c_{2}^{i=1}=1, c_{1}^{i=i_{\max }}=0.5$ and $c_{2}^{i=i_{\max }}=0$, and call out the needed information for intended test system, such as $m, V, \mathrm{C}, W, w_{\mathrm{d}}, v_{d}, \alpha_{d}$ and $\beta_{d}$,for all subsystems (for $d=1: m)$.

Step 2: Produce the initial random population matrix $(N \times 2 * m)$ using the minimum and maximum limits of the variables.

Step 3: Calculate the objective function $f(r, n)$ of RRAP by imposing the non-linear constraints $g_{1}(r, n), \quad g_{2}(r, n)$ and $g_{3}(r, n)$ for every available solution in the initial population of the LJaya-TVAC algorithm.

Step 4: Produce the new population of LJaya-TVAC algorithm using (15) (the first phase).

Step 5: Calculate the objective function $f(r, n)$ of RRAP by imposing the non-linear constraints $g_{1}(r, n), g_{2}(r, n)$ and $g_{3}(r, n)$ for the generated population in Step 4 .

Step 6: Produce the new population of the LJaya-TVAC algorithm using (18) (the second phase).

Step 7: Calculate the objective function $f(r, n)$ of RRAP by imposing the non-linear constraints $g_{1}(r, n), g_{2}(r, n)$ and $g_{3}(r, n)$ for the generated population in Step 6 . 
Step 8: Repeat Steps 4-7 till (for $i=1: i_{\max }$ ) reaching the maximum number of iterations.

The best results obtained from the proposed LJaya-TVAC algorithm compared with previously reported results for RRAPs for five test systems over 30 runs with $i_{\max }=d^{*} 1000$, and the population size of $N=4 * d,(d=2 * m)$, are summarized in Tables 2-6. The best solution values are in bold including $f(r, n)$ (the obtained best value of the objective function), Mean (the mean value of the best results), Worst (the obtained worst value of the objective function), Std (the standard deviation of the best results) indexes and the maximum possible improvement (MPI) index (dos Santos Coelho 2009). The LJaya-TVAC algorithm is compared with previously reported best results (He et al. 2015). The MPI index is defined as follows (Valian et al. 2013):

$\operatorname{MPI}(\%)=\frac{f_{\text {Laya-TVAC }}(r, n)-f_{\text {other }}(r, n)}{1-f_{\text {other }}(r, n)}$,

$f_{\text {LJaya-TVAC }}(r, n)$ : the best results obtain by LJaya-TVAC algorithm,

$f_{\text {other }}(r, n):$ the best results reported by previous studies.

Also, slack $(g)=l-g$, for example slack $\left(g_{1}\right)=V-g_{1}(r, n)$.

The best result of the series test system by LJaya-TVAC algorithm shown in Table 2 is 0.931682388 which is compared with previously reported results including PSSO (Huang 2015), IA (Chen 2006), IPSO (Wu et al. 2011), a new IA (NIA) (Hsieh and You 2011), ARICA (Afonso, Mariani, and dos Santos Coelho 2013), and Improved Cuckoo Search ICS (Valian et al. 2013). The proposed LJaya-TVAC algorithm obtains the best solutions among all the solutions including $f_{1}(r, n)$, Mean, Worst and Std indexes as shown in Table 2, with the obtained results of $0.931682386,0.9316823797$ and $8.15 \mathrm{e}-22$ respectively. It can be seen from Table 2 that, for PSSO (Huang 2015), IA (Chen 2006), IPSO (Wu et al. 2011), NIA (Hsieh and You 2011), AR-ICA (Afonso, Mariani, and dos Santos Coelho 2013), ICS (Valian 
et al. 2013), the corresponding improvements (MPI) made by the LJaya-TVAC algorithm are $1.288 \mathrm{e}-4,6.423 \mathrm{e}-3,3.554 \mathrm{e}-3,7.026 \mathrm{e}-5,4.388 \mathrm{e}-2$, and $1.464 \mathrm{e}-6$, respectively.

Table 2. Comparison of the best results obtained by LJaya-TVAC with some of the previously reported results for the series test system.

\begin{tabular}{|c|c|c|c|c|c|c|c|}
\hline Parameter & PSSO & IA & IPSO & NIA & AR-ICA & ICS & LJaya-TVAC \\
\hline$r_{1}$ & 0.77946645 & 0.779266 & 0.78037307 & 0.779462304 & 0.779874 & 0.779416938 & 0.779402388 \\
\hline$r_{2}$ & 0.87173278 & 0.872513 & 0.87178343 & 0.871883456 & 0.872057 & 0.871833278 & 0.871835465 \\
\hline$r_{3}$ & 0.90284951 & 0.902634 & 0.90240890 & 0.902800879 & 0.903426 & 0.902885082 & 0.902882077 \\
\hline$r_{4}$ & 0.71148780 & 0.710648 & 0.71147356 & 0.711350168 & 0.710960 & 0.711393868 & 0.711408035 \\
\hline$r_{5}$ & 0.78781644 & 0.788406 & 0.78738760 & 0.787861587 & 0.786902 & 0.787803712 & 0.787793007 \\
\hline$\overline{n_{1}}$ & 3 & 3 & 3 & 3 & 3 & 3 & 3 \\
\hline$n_{2}$ & 2 & 2 & 2 & 2 & 2 & 2 & 2 \\
\hline$n_{3}$ & 2 & 2 & 2 & 2 & 2 & 2 & 2 \\
\hline$n_{4}$ & 3 & 3 & 3 & 3 & 3 & 3 & 3 \\
\hline$n_{5}$ & 3 & 3 & 3 & 3 & 3 & 3 & 3 \\
\hline$f_{1}(r, n)$ & 0.93168230 & 0.931678 & 0.93167996 & 0.93168234 & 0.93167939 & 0.931682387 & 0.931682388 \\
\hline Slack $\left(g_{1}\right)$ & - & 27 & 27 & 27 & 27 & 27 & 27 \\
\hline Slack $\left(g_{2}\right)$ & - & 0.001559 & 0.000101 & 0.0000005284 & 0.000099 & 0.000000265 & $2.19 \mathrm{e}-08$ \\
\hline Slack $\left(g_{3}\right)$ & - & 7.518918 & 7.518918 & 7.518918 & 7.518918 & 7.518918241 & 7.51891824 \\
\hline MPI (\%) & $1.288 \mathrm{e}-4$ & $6.423 \mathrm{e}-3$ & $3.554 \mathrm{e}-3$ & $7.026 \mathrm{e}-5$ & $4.388 \mathrm{e}-2$ & $1.464 \mathrm{e}-6$ & - \\
\hline Mean & 0.871793835 & - & 0.92847132 & 0.93168222 & 0.92182324 & 0.92987132 & 0.931682386 \\
\hline Worst & 0.64815102 & - & 0.91011333 & - & 0.82989353 & 0.92066034 & 0.9316823797 \\
\hline Std & 0.055331848 & - & $5.2382 \mathrm{e}-03$ & $1.3 \mathrm{e}-14$ & 0.01863188 & $1.99046 \mathrm{e}-03$ & $8.15 \mathrm{e}-22$ \\
\hline
\end{tabular}

The best results for the series-parallel test system by LJaya-TVAC algorithm compared with ICS (Valian et al. 2013), MPSO (Liu and Qin 2014), IPSO (Wu et al. 2011), NIA (Hsieh and You 2011), AR-ICA (Afonso, Mariani, and dos Santos Coelho 2013), NAFSA (He et al. 2015) are shown in Table 3. The MPSO, NAFSA and proposed LJaya-TVAC obtain the better results than the other algorithms. The best values obtained by ICS, MPSO, IPSO, NIA, AR-ICA, NAFSA are 0.999976649, 0.9999766491, 0.99997664, 0.999976649, 0.99997661 
and0.9999766491 respectively. Also, LJaya-TVAC obtains the better indexes in terms of Mean, Worst and Std than all other algorithms, with the obtained results of 0.9999766491, 0.99997664904 and $8.15 \mathrm{e}-25$ respectively.

Table 3. Comparison of the best results obtained by LJaya-TVAC with some of the previously reported results for the series-parallel test system.

\begin{tabular}{|c|c|c|c|c|c|c|c|}
\hline $\begin{array}{c}\text { Paramete } \\
\text { r }\end{array}$ & ICS & MPSO & IPSO & NIA & AR-ICA & NAFSA & LJaya-TVAC \\
\hline$r_{1}$ & 0.819927087 & 0.8196547522 & 0.81918526 & 0.819591561 & 0.82201264 & 0.819737753 & 0.819659132 \\
\hline$r_{2}$ & 0.845267657 & 0.8449752789 & 0.84366421 & 0.844951068 & 0.84365640 & 0.844991099 & 0.844980808 \\
\hline$r_{3}$ & 0.895491554 & 0.8955087772 & 0.89472992 & 0.895428548 & 0.89129092 & 0.895529543 & 0.895506189 \\
\hline$r_{4}$ & 0.895440692 & 0.8955091117 & 0.89537628 & 0.895522339 & 0.89869886 & 0.895433687 & 0.895506537 \\
\hline$r_{5}$ & 0.868318775 & 0.8684491638 & 0.86912724 & 0.868490229 & 0.86824939 & 0.868434824 & 0.868447819 \\
\hline$n_{1}$ & 2 & 2 & 2 & 2 & 2 & 2 & 2 \\
\hline$n_{2}$ & 2 & 2 & 2 & 2 & 2 & 2 & 2 \\
\hline$n_{3}$ & 2 & 2 & 2 & 2 & 2 & 2 & 2 \\
\hline$n_{4}$ & 2 & 2 & 2 & 2 & 2 & 2 & 2 \\
\hline$n_{5}$ & 4 & 4 & 4 & 4 & 4 & 4 & 4 \\
\hline$f_{2}(r, n)$ & 0.999976649 & 0.9999766491 & 0.99997664 & 0.999976649 & 0.99997661 & 0.9999766491 & 0.9999766491 \\
\hline Slack $\left(g_{1}\right)$ & 40 & 40 & 40 & 40 & 40 & 40 & 40 \\
\hline Slack $\left(g_{2}\right)$ & 0.0000161 & $8.4 \mathrm{e}-9$ & 0.000561 & 0.0 & 0.000396 & $1.39152 \mathrm{e}-10$ & $7.959 \mathrm{e}-010$ \\
\hline Slack $\left(g_{3}\right)$ & 1.6092890 & 1.6092889667 & 1.609289 & 1.609289 & 1.609289 & 1.609288966 & 1.6092889667 \\
\hline MPI (\%) & $4.282 \mathrm{e}-04$ & $\mathbf{0}$ & $3.896 \mathrm{e}-02$ & $4.282 \mathrm{e}-04$ & $1.672 \mathrm{e}-01$ & 0 & - \\
\hline Mean & 0.99997090 & 0.9999766174 & 0.99996974 & 0.999976649 & 0.99994991 & 0.9999766490 & 0.9999766491 \\
\hline Worst & 0.99994886 & 0.9999765280 & 0.99994106 & - & 0.99984762 & - & 0.99997664904 \\
\hline Std & $4.45 \mathrm{e}-06$ & $3.87 \mathrm{e}-08$ & $1.336 \mathrm{e}-05$ & $3.0 \mathrm{e}-21$ & $2.58 \mathrm{e}-06$ & $3.18206 \mathrm{e}-10$ & $8.15 \mathrm{e}-25$ \\
\hline
\end{tabular}

Also, the best results for the complex (bridge) test system and overspeed protection system by different algorithms are summarized in Tables 4-5. The comparison of the best results shows that the proposed LJaya-TVAC obtains the better results than all the other algorithms. It can be seen from Table 4 for complex (bridge) test system that, for IA (Chen 2006), EGHS 
(Zou et al. 2011), IPSO (Wu et al. 2011), AR-ICA (Afonso, Mariani, and dos Santos Coelho 2013), PSFS (Mellal and Zio 2016), NAFSA (He et al. 2015), the corresponding improvements (MPI) made by the LJaya-TVAC algorithm are 3.858e-01, 3.398e-02, 6.815e$03,6.815 \mathrm{e}-03,1.087 \mathrm{e}-05$, and $1.370 \mathrm{e}-03$, respectively.

Also, it can be seen from Table 5 for the overspeed protection system test system that, for IA (Chen 2006), EGHS (Zou et al. 2011), PSSO (Huang 2015), AR-ICA (Afonso, Mariani, and dos Santos Coelho 2013), NAFSA (He et al. 2015), GA-PSO (Sheikhalishahi et al. 2013), the corresponding improvements (MPI) made by the LJaya-TVAC algorithm are $21.853,9.847 \mathrm{e}-$ 02, 1.03e-02, 3.699e-03, 1.496e-05, and 1.03e-02, respectively.

Table 4. Comparison of the best results obtained by LJaya-TVAC with some of the previously reported results for the complex (bridge) test system.

\begin{tabular}{|c|c|c|c|c|c|c|c|}
\hline Parameter & IA & EGHS & IPSO & AR-ICA & PSFS & NAFSA & LJaya-TVAC \\
\hline$r_{1}$ & 0.812485 & 0.82983999 & 0.82868361 & 0.82764257 & 0.82812141729 & 0.82832179189 & 0.828081997 \\
\hline$r_{2}$ & 0.867661 & 0.85798911 & 0.85802567 & 0.85747845 & 0.85781341076 & 0.85797450730 & 0.857823532 \\
\hline$r_{3}$ & 0.861221 & 0.91333926 & 0.91364616 & 0.91419677 & 0.91423927822 & 0.91422098825 & 0.914227868 \\
\hline$r_{4}$ & 0.713852 & 0.64674479 & 0.64803407 & 0.64927379 & 0.64807680660 & 0.64775717018 & 0.648117404 \\
\hline$r_{5}$ & 0.756699 & 0.70310972 & 0.70227595 & 0.70409200 & 0.70424641245 & 0.70300666185 & 0.70436276 \\
\hline$\overline{n_{1}}$ & 3 & 3 & 3 & 3 & 3 & 3 & 3 \\
\hline$n_{2}$ & 3 & 3 & 3 & 3 & 3 & 3 & 3 \\
\hline$n_{3}$ & 3 & 2 & 2 & 2 & 2 & 2 & 2 \\
\hline$\overline{n 4}$ & 3 & 4 & 4 & 4 & 4 & 4 & 4 \\
\hline$n_{5}$ & 1 & 1 & 1 & 1 & 1 & 1 & 1 \\
\hline$f_{3}(r, n)$ & 0.99988921 & 0.99988960 & 0.99988963 & 0.99988963 & 0.99988963751 & 0.99988963601 & 0.999889637522 \\
\hline Slack $\left(g_{1}\right)$ & 19 & 5 & 5 & 5 & 5 & 5 & 5 \\
\hline Slack $\left(g_{2}\right)$ & 0.001494 & 0.00000594 & 0.00000359 & 0.00004428 & $2.85464 \mathrm{e}-6$ & $1.5485 \mathrm{e}-5$ & $2.960 \mathrm{e}-06$ \\
\hline Slack $\left(g_{3}\right)$ & 4.264770 & 1.56046629 & 1.56046629 & 1.56046629 & 1.560466288 & 1.56046629 & 1.560466288 \\
\hline MPI (\%) & $3.858 \mathrm{e}-01$ & $3.398 \mathrm{e}-02$ & $6.815 e-03$ & $6.815 \mathrm{e}-03$ & $1.087 \mathrm{e}-05$ & $1.370 \mathrm{e}-03$ & - \\
\hline Mean & - & 0.99988263 & 0.99988799 & 0.99979532 & - & 0.99987756441 & 0.99988963752 \\
\hline Worst & - & 0.99982887 & 0.99970178 & 0.99939296 & - & - & 0.999889637513 \\
\hline
\end{tabular}




\begin{tabular}{|c|c|c|c|c|c|c|c|}
\hline Std & - & $1.6 \mathrm{e}-05$ & $4.0163 \mathrm{e}-05$ & $1.037 \mathrm{e}-04$ & $\mathbf{3 . 7 e - 0 2 3}$ & $2.1017 \mathrm{e}-05$ & $8.16 \mathrm{e}-020$ \\
\hline
\end{tabular}

Table 5. Comparison of the best results obtained by LJaya-TVAC with some of the previously

\begin{tabular}{|c|c|c|c|c|c|c|c|}
\hline Parameter & IA & EGHS & PSSO & AR-ICA & NAFSA & GA-PSO & LJaya-TVAC \\
\hline$r_{1}$ & 0.903800 & 0.900925066 & 0.90166461 & 0.90148988 & 0.90160779120 & 0.901628 & 0.901614807 \\
\hline$r_{2}$ & 0.874992 & 0.851636929 & 0.88817296 & 0.85003526 & 0.84993077684 & 0.888230 & 0.849921181 \\
\hline$r_{3}$ & 0.919898 & 0.948079849 & 0.94821033 & 0.94812952 & 0.94814603278 & 0.948121 & 0.948141393 \\
\hline$r_{4}$ & 0.890609 & 0.887654500 & 0.84987084 & 0.88823833 & 0.88821809379 & 0.849921 & 0.888222817 \\
\hline$n_{1}$ & 5 & 5 & 5 & 5 & 5 & 5 & 5 \\
\hline$n_{2}$ & 5 & 6 & 5 & 5 & 6 & 5 & 6 \\
\hline$n_{3}$ & 5 & 4 & 4 & 4 & 4 & 4 & 4 \\
\hline$n_{4}$ & 5 & 5 & 6 & 5 & 6 & 6 & 5 \\
\hline$f_{4}(r, n)$ & 0.999942 & 0.99995463 & 0.99995467 & 0.999954673 & 0.99995467467 & 0.99995467 & $\mathbf{0 . 9 9 9 9 5 4 6 7 4 6 7 6 7 8 2}$ \\
\hline Slack $\left(g_{1}\right)$ & 50 & 55 & - & 55 & 55 & 55 & 55 \\
\hline Slack $\left(g_{2}\right)$ & 0.002152 & 0.00000105 & - & 0.00213782 & $4.5195 \mathrm{e}-07$ & 0.000006 & $1.614 \mathrm{e}-10$ \\
\hline Slack $\left(g_{3}\right)$ & 28.803701 & 24.80188272 & - & 24.8018827 & 24.802 & 15.363463 & 24.8018827 \\
\hline MPI $(\%)$ & $\mathbf{2 1 . 8 5 3}$ & $\mathbf{9 . 8 4 7 e - 0 2}$ & $\mathbf{1 . 0 3 e - 0 2}$ & $\mathbf{3 . 6 9 9 e - 0 3}$ & $\mathbf{1 . 4 9 6 e - 0 5}$ & $\mathbf{1 . 0 3 e - 0 2}$ & - \\
\hline Mean & - & 0.99993588 & 0.9999416669 & 0.99993804 & 0.99995075542 & 0.99995467 & $\mathbf{0 . 9 9 9 9 5 4 6 7 4 6 7 6 7 8}$ \\
\hline Worst & - & 0.99985315 & 0.99986938 & 0.99982276 & - & 0.99995467 & $\mathbf{0 . 9 9 9 9 5 4 6 7 4 6 7 6 7 7 8}$ \\
\hline Std & - & $2.2 \mathrm{e}-05$ & $1.61 \mathrm{e}-5$ & 0.00002204 & $4.43 \mathrm{e}-06$ & $1.0 \mathrm{e}-16$ & $\mathbf{4 . 8 6 e - 3 2}$ \\
\hline
\end{tabular}

Table 6 shows that the best solution for a large-scale test system is related to the proposed LJaya-TVAC which is compared with the solution reported by PSFS (Mellal and Zio 2016). The best results are 0.89051730902 and 0.891136424677689 by PSFS (Mellal and Zio 2016) and the proposed algorithm, respectively, and the result provided by the LJaya-TVAC is better than PSFS (Mellal and Zio 2016). The results show that the LJaya-TVAC algorithm is very reliable for the real large-scale optimization problems. In Table 6, for the best result obtain by the PSFS (Mellal and Zio 2016), the corresponding improvement (MPI) made by the LJaya-TVAC algorithm is 5.655e-01. 
Table 6. Comparison of the best results obtained by LJaya-TVAC with some of the previously reported results for the large-scale test system.

\begin{tabular}{|c|c|c|c|c|c|}
\hline Parameter & PSFS & LJaya-TVAC & Parameter & PSFS & LJaya-TVAC \\
\hline$r_{1}$ & 0.920682125899 & 0.921084976 & $n_{1}$ & 2 & 2 \\
\hline$r_{2}$ & 0.952579760087 & 0.952404213 & $n_{2}$ & 2 & 2 \\
\hline$r_{3}$ & 0.840370879766 & 0.841155866 & $n_{3}$ & 3 & 3 \\
\hline$r_{4}$ & 0.934499487329 & 0.935072836 & $n_{4}$ & 2 & 2 \\
\hline$r_{5}$ & 0.806884188682 & 0.807515426 & $n_{5}$ & 3 & 3 \\
\hline$r_{6}$ & 0.895206390582 & 0.894297746 & $n_{6}$ & 2 & 2 \\
\hline$r_{7}$ & 0.811801524606 & 0.81181724 & $n_{7}$ & 3 & 3 \\
\hline$r_{8}$ & 0.814181963158 & 0.813453019 & $n_{8}$ & 3 & 3 \\
\hline$r_{9}$ & 0.836220223575 & 0.901645065 & $n_{9}$ & 3 & 2 \\
\hline$r_{10}$ & 0.827983638973 & 0.825833294 & $\overline{n_{10}}$ & 3 & 3 \\
\hline$r_{11}$ & 0.814585208335 & 0.815100918 & $n_{11}$ & 3 & 3 \\
\hline$r_{12}$ & 0.837346324449 & 0.838160321 & $\overline{n_{12}}$ & 3 & 3 \\
\hline$r_{13}$ & 0.841065088128 & 0.841043767 & $n_{13}$ & 3 & 3 \\
\hline$r_{14}$ & 0.821075460589 & 0.820672214 & $n_{14}$ & 3 & 3 \\
\hline$r_{15}$ & 0.850117124276 & 0.85096942 & $n_{15}$ & 3 & 3 \\
\hline$r_{16}$ & 0.838491056144 & 0.838425251 & $n_{16}$ & 3 & 3 \\
\hline$r_{17}$ & 0.823073269011 & 0.824306984 & $n_{17}$ & 3 & 3 \\
\hline$r_{18}$ & 0.809956458845 & 0.810315221 & $n_{18}$ & 3 & 3 \\
\hline$r_{19}$ & 0.807719250830 & 0.808690588 & $\overline{n_{19}}$ & 3 & 3 \\
\hline$R_{20}$ & 0.897948276899 & 0.833344431 & $n_{20}$ & 2 & 3 \\
\hline Slack $\left(g_{1}\right)$ & 143 & 158 & $f_{5}(r, n)$ & 0.89051730902 & 0.891136424677689 \\
\hline Slack $\left(g_{2}\right)$ & 0.00275018 & $1.453186 \mathrm{e}-04$ & Mean & - & 0.888020914198434 \\
\hline Slack $\left(g_{3}\right)$ & 2.1970797 & 2.197079756 & Std & $3.5 \mathrm{e}-08$ & $7.15 \mathrm{e}-12$ \\
\hline Worst & - & 0.886359901275289 & MPI (\%) & $5.655 \mathrm{e}-01$ & - \\
\hline
\end{tabular}

It is obvious from Tables 1-6 that the proposed LJaya-TVAC algorithm can be useful and effective for optimization problems of engineering systems in comparison with the original Jaya algorithm and the best results reported in the recent literature by other algorithms. 


\section{Conclusion}

In this paper, a hybrid enhanced Jaya algorithm based on TVAC and learning phase, called LJaya-TVAC has been proposed to efficiently solve various types of nonlinear mixed-integer RRAPs. The series, series-parallel, complex (bridge) and overspeed protection systems have been considered as RRAPs. The effectiveness of proposed LJaya-TVAC algorithm to achieve the optimal solutions of the standard real-parameter uni-modal and multi-model benchmark, as well as various RRAPs, was tested and compared with original Jaya algorithm and other optimal solutions reported in the recent literature.

In RRAPs, in addition to the reliability objective function, some other objective functions can be considered such as the overall cost (Ardakan, Hamadani, and Alinaghian 2015). The obtained optimal results of the paper have provided evidence for better and effective optimization performance of the proposed LJaya-TVAC algorithm in comparison with optimal solutions reported in the recent literature. Note that although the proposed algorithm is very reliable for the large-scale optimization problems in practice which is successfully tested for different systems in the paper, it adds some complexity to the simple Java algorithm. Addressing this problem is beyond the scope of this paper and would be the focus of our future work. Also in the future work, the proposed LJaya-TVAC algorithm can be considered for multi-objective optimization problems and compared with other algorithms such as NSGA-II and so on in the literature.

\section{References}

Afonso, Leonardo Dallegrave, Viviana Cocco Mariani, and Leandro dos Santos Coelho. 2013. "Modified imperialist competitive algorithm based on attraction and repulsion concepts for reliability-redundancy optimization." Expert Systems with Applications 40 (9):3794-802.

Ardakan Abouei, Mostafa, Mohammad Sima, Ali Zeinal Hamadani, and David W Coit. 2016. "A novel strategy for redundant components in reliability-redundancy allocation problems." IIE Transactions 48(11): 1043-1057. 
Ardakan, Mostafa Abouei, and Ali Zeinal Hamadani. 2014a. "Reliability-redundancy allocation problem with cold-standby redundancy strategy." Simulation Modelling Practice and Theory 42:107-18.

Ardakan, Mostafa Abouei, and Ali Zeinal Hamadani. 2014b. "Reliability optimization of seriesparallel systems with mixed redundancy strategy in subsystems." Reliability Engineering \& System Safety 130:132-9.

Ardakan, Mostafa Abouei, Ali Zeinal Hamadani, and Mehdi Alinaghian. 2015. "Optimizing biobjective redundancy allocation problem with a mixed redundancy strategy." ISA transactions 55:116-28.

Chatwattanasiri, Nida, David W Coit, and Naruemon Wattanapongsakorn. 2016. "System redundancy optimization with uncertain stress-based component reliability: Minimization of regret." Reliability Engineering \& System Safety 154:73-83.

Chen, Ta-Cheng. 2006. "IAs based approach for reliability redundancy allocation problems." Applied Mathematics and Computation 182 (2):1556-67.

Chen, Ta-Cheng, and Peng-Sheng You. 2005. "Immune algorithms-based approach for redundant reliability problems with multiple component choices." Computers in Industry 56 (2):195205.

Dohi, Tadashi, Naoto Kaio, Won Young, Ho-Gyun Kim, Chang-Ok Bae, and Dong-Jun Park. 2006. "Reliability-redundancy optimization using simulated annealing algorithms." Journal of Quality in Maintenance Engineering 12 (4):354-63.

dos Santos Coelho, Leandro. 2009. "An efficient particle swarm approach for mixed-integer programming in reliability-redundancy optimization applications." Reliability Engineering \& System Safety 94 (4):830-7.

dos Santos Coelho, Leandro, L de A Diego, and Viviana Cocco Mariani. 2011. "ReliabilityRedundancy Optimization Using a Chaotic Differential Harmony Search Algorithm." In Handbook of Swarm Intelligence, 503-16. Springer.

Feizollahi, Mohammad Javad, Roya Soltani, and Hadi Feyzollahi. 2015. "The robust cold standby redundancy allocation in series-parallel systems with budgeted uncertainty." IEEE Transactions on Reliability 64 (2):799-806.

Hamadani, Ali Zeinal, Mostafa Abouei Ardakan, Taghi Rezvan, and Mohammad Mehran Honarmandian. 2013. "Location-allocation problem for intra-transportation system in a big company by using meta-heuristic algorithm." Socio-Economic Planning Sciences 47 (4):30917.

He, Pan, Kaigui Wu, Jie Xu, Junhao Wen, and Zhuo Jiang. 2013. "Multilevel redundancy allocation using two dimensional arrays encoding and hybrid genetic algorithm." Computers \& Industrial Engineering 64 (1):69-83.

He, Qiang, Xiangtao Hu, Hong Ren, and Hongqi Zhang. 2015. "A novel artificial fish swarm algorithm for solving large-scale reliability-redundancy application problem." ISA transactions 59:105-13.

Hsieh, Y-C, and P-S You. 2011. "An effective immune based two-phase approach for the optimal reliability-redundancy allocation problem." Applied Mathematics and Computation 218 (4):1297-307.

Hsieh, Yi-Chih, Ta-Cheng Chen, and Dennis L Bricker. 1998. "Genetic algorithms for reliability design problems." Microelectronics Reliability 38 (10):1599-605.

Huang, Chia-Ling. 2015. "A particle-based simplified swarm optimization algorithm for reliability redundancy allocation problems." Reliability Engineering \& System Safety 142:221-30.

Jang, Kil-Woong, and Jae-Hwan Kim. 2011. "A tabu search for multiple multi-level redundancy allocation problem in series-parallel systems." International Journal of Industrial Engineering: Theory, Applications and Practice 18 (3).

Li, Zhaojun, Mohammadsadegh Mobin, and Thomas Keyser. 2016. "Multi-Objective and Multi-Stage Reliability Growth Planning in Early Product-Development Stage." IEEE Transactions on Reliability 65 (2):769-81.

Liang, Yun-Chia, and Alice E Smith. 2004. "An ant colony optimization algorithm for the redundancy allocation problem (RAP)." IEEE Transactions on Reliability 53 (3):417-23. 
Liu, Gia-Shie. 2006. "A combination method for reliability-redundancy optimization." Engineering Optimization 38 (04):485-99.

Liu, Yubao. 2016. "Improved Bat Algorithm for Reliability-Redundancy Allocation Problems." International Journal of Security and Its Applications 10 (2):1-12.

Liu, Yubao, and Guihe Qin. 2014. "A hybrid TS-DE algorithm for reliability redundancy optimization problem." Journal of Computers 9 (9):2050-7.

Mellal, Mohamed Arezki, and Enrico Zio. 2016. "A penalty guided stochastic fractal search approach for system reliability optimization." Reliability Engineering \& System Safety 152:213-27.

Ouzineb, Mohamed, Mustapha Nourelfath, and Michel Gendreau. 2011. "A heuristic method for nonhomogeneous redundancy optimization of series-parallel multi-state systems." Journal of Heuristics 17 (1):1-22.

Pourdarvish, Ahmad, and Zahra Ramezani. 2013. "Cold standby redundancy allocation in a multilevel series system by memetic algorithm." International Journal of Reliability, Quality and Safety Engineering 20 (03):1340007.

Rao, R. 2016. "Jaya: A simple and new optimization algorithm for solving constrained and unconstrained optimization problems." International Journal of Industrial Engineering Computations 7 (1):19-34.

Rao, R, and Vivek Patel. 2012. "An elitist teaching-learning-based optimization algorithm for solving complex constrained optimization problems." International Journal of Industrial Engineering Computations 3 (4):535-60.

Rao, R Venkata. 2015. Teaching Learning Based Optimization Algorithm: And Its Engineering Applications: Springer.

Rao, R Venkata, Dhiraj P Rai, and Joze Balic. 2016. "Surface Grinding Process Optimization Using Jaya Algorithm." In Computational Intelligence in Data Mining, Springer 487-495

Rao, Ravipudi V, Vimal J Savsani, and DP Vakharia. 2011. "Teaching-learning-based optimization: a novel method for constrained mechanical design optimization problems." Computer-Aided Design 43 (3):303-15.

Rao, RV, KC More, J Taler, and P Ocłoń. 2016. "Dimensional optimization of a micro-channel heat sink using Jaya algorithm." Applied Thermal Engineering 103:572-82.

Ratnaweera, Asanga, Saman K Halgamuge, and Harry C Watson. 2004. "Self-organizing hierarchical particle swarm optimizer with time-varying acceleration coefficients." IEEE Transactions on evolutionary computation 8 (3):240-55.

Sadjadi, Seyed Jafar, and Roya Soltani. 2012. "Alternative design redundancy allocation using an efficient heuristic and a honey bee mating algorithm." Expert Systems with Applications 39 (1):990-9.

Sheikhalishahi, M, V Ebrahimipour, H Shiri, H Zaman, and M Jeihoonian. 2013. "A hybrid GA-PSO approach for reliability optimization in redundancy allocation problem." The International Journal of Advanced Manufacturing Technology 68 (1-4):317-38.

Soltani, Roya. 2014. "Reliability optimization of binary state non-repairable systems: A state of the art survey." International Journal of Industrial Engineering Computations 5 (3):339.

Soltani, Roya, Seyed Jafar Sadjadi, and Reza Tavakkoli-Moghaddam. 2015. "Entropy based redundancy allocation in series-parallel systems with choices of a redundancy strategy and component type: A multi-objective model." Appl. Math 9 (2):1049-58.

Suganthan, Ponnuthurai N, Nikolaus Hansen, Jing J Liang, Kalyanmoy Deb, Ying-Ping Chen, Anne Auger, and Santosh Tiwari. 2005. "Problem definitions and evaluation criteria for the CEC 2005 special session on real-parameter optimization." KanGAL report.

Suman, Balram. 2003. "Simulated annealing-based multiobjective algorithms and their application for system reliability." Engineering Optimization 35 (4):391-416.

Tan, Yue, Guan-zheng Tan, and Shu-guang Deng. 2013. "Hybrid particle swarm optimization with differential evolution and chaotic local search to solve reliability-redundancy allocation problems." Journal of Central South University 20:1572-81.

Valian, Ehsan, Saeed Tavakoli, Shahram Mohanna, and Atiyeh Haghi. 2013. "Improved cuckoo search for reliability optimization problems." Computers \& Industrial Engineering 64 (1):459-68. 
Valian, Ehsan, and Elham Valian. 2013. "A cuckoo search algorithm by Lévy flights for solving reliability redundancy allocation problems." Engineering Optimization 45 (11):1273-86.

Venkata Rao, R, and GG Waghmare. 2017. "A new optimization algorithm for solving complex constrained design optimization problems." Engineering Optimization 49 (1): 60-83.

Wu, Peifeng, Liqun Gao, Dexuan Zou, and Steven Li. 2011. "An improved particle swarm optimization algorithm for reliability problems." ISA transactions 50 (1):71-81.

Yeh, Wei-Chang, and Tsung-Jung Hsieh. 2011. "Solving reliability redundancy allocation problems using an artificial bee colony algorithm." Computers \& Operations Research 38 (11):146573.

Zhang, HongQi, XiangTao Hu, XiaoDong Shao, ZiCheng Li, and YuHui Wang. 2013. "IPSO-based hybrid approaches for reliability-redundancy allocation problems." Science China Technological Sciences 56 (11):2854-64.

Zou, Dexuan, Liqun Gao, Steven Li, and Jianhua Wu. 2011. "An effective global harmony search algorithm for reliability problems." Expert Systems with Applications 38 (4):4642-8.

Zou, Dexuan, Liqun Gao, Jianhua Wu, Steven Li, and Yang Li. 2010. "A novel global harmony search algorithm for reliability problems." Computers \& Industrial Engineering 58 (2):30716.

Appendix. Data of the test systems.

Table A.1. Data of the series system $(P 1)$.

\begin{tabular}{|c|c|c|c|c|c|c|c|}
\hline Stage & $10^{5} \times \alpha_{d}$ & $\beta_{d}$ & $w_{d} v_{d}^{2}$ & $w_{d}$ & V & C & $W$ \\
\hline 1 & 1.0 & 1.5 & 1 & 6 & \multirow[t]{4}{*}{250} & \multirow[t]{4}{*}{400} & \multirow[t]{4}{*}{500} \\
\hline 2 & 2.3 & 1.5 & 2 & 6 & & & \\
\hline 3 & 0.3 & 1.5 & 3 & 8 & & & \\
\hline 4 & 2.3 & 1.5 & 2 & 7 & & & \\
\hline
\end{tabular}

Table A.2. Data of the series-parallel system (P2).

\begin{tabular}{|c|c|c|c|c|c|c|c|}
\hline Stage & $10^{5} \times \alpha_{d}$ & $\beta_{d}$ & $w_{d} v_{d}^{2}$ & $w_{d}$ & $V$ & $C$ & $W$ \\
\hline 1 & 2.500 & 1.5 & 2 & 3.5 & \multirow[t]{5}{*}{180} & \multirow[t]{5}{*}{175} & \multirow[t]{5}{*}{100} \\
\hline 2 & 1.450 & 1.5 & 4 & 4.0 & & & \\
\hline 3 & 0.541 & 1.5 & 5 & 4.0 & & & \\
\hline 4 & 0.541 & 1.5 & 8 & 3.5 & & & \\
\hline 5 & 2.100 & 1.5 & 4 & 4.5 & & & \\
\hline
\end{tabular}


Table A.3. Data of the complex (bridge) system (P3).

\begin{tabular}{|c|c|c|c|c|c|c|c|}
\hline Stage & $10^{5} \times \alpha_{d}$ & $\beta$ & $w_{d} v_{d}^{2}$ & $w_{d}$ & $V$ & $C$ & $W$ \\
\hline 1 & 2.330 & 1.5 & 1 & 7 & \multirow[t]{5}{*}{180} & \multirow[t]{5}{*}{175} & \multirow[t]{5}{*}{200} \\
\hline 2 & 1.450 & 1.5 & 2 & 8 & & & \\
\hline 3 & 0.541 & 1.5 & 3 & 8 & & & \\
\hline 4 & 8.050 & 1.5 & 4 & 6 & & & \\
\hline 5 & 1.950 & 1.5 & 2 & 9 & & & \\
\hline
\end{tabular}

Table A.4. Data of the fourth test system (P4).

\begin{tabular}{|c|c|c|c|c|c|c|c|c|}
\hline Stage & $10^{5} \times \alpha_{d}$ & $\beta_{d}$ & $w_{d} v_{d}^{2}$ & $w_{d}$ & $V$ & $C$ & $W$ & $T$ \\
\hline 1 & 1.0 & 1.5 & 1 & 6 & \multirow[t]{4}{*}{250} & \multirow[t]{4}{*}{400} & \multirow[t]{4}{*}{500} & \multirow[t]{4}{*}{$1000 \mathrm{~h}$} \\
\hline 2 & 2.3 & 1.5 & 2 & 6 & & & & \\
\hline 3 & 0.3 & 1.5 & 3 & 8 & & & & \\
\hline 4 & 2.3 & 1.5 & 2 & 7 & & & & \\
\hline
\end{tabular}

